\title{
Host-associated genetic differentiation in Thrips tabaci (Insecta; Thysanoptera), as determined from mtDNA sequence data
}

\author{
PC Brunner ${ }^{1}$, EK Chatzivassiliou ${ }^{2}$, NI Katis ${ }^{2}$ and JE Frey ${ }^{1}$ \\ ${ }^{1}$ Agroscope FAW Wädenswil, Swiss Federal Research Station for Horticulture, PO Box 185, Wädenswil CH-8820, Switzerland; ${ }^{2}$ Plant \\ Pathology Laboratory, Aristotle University of Thessaloniki, Faculty of Agriculture, PO Box 269, Thessaloniki 54124, Greece
}

\begin{abstract}
We tested for host-associated genetic differentiation in 22 populations of Thrips tabaci collected from tobacco and leek, respectively. Clustering analyses and haplotype networks based on sequence variation at a fragment of the mitochondrial cytochrome oxidase I gene yielded three major evolutionary lineages; two were clearly associated with leek and the third with tobacco. These genetic findings corroborated recent experimental observations on the heterogeneity of $T$. tabaci populations with regard to host-plant preference and their capacity to be vectors for tomato spotted wilt virus.
\end{abstract}

Estimated divergence times suggested an ancient divergence of these lineages dating back to the Miocene 28-21 million years ago. $F_{\mathrm{ST}}$ values between these lineages ranged between 0.824 and 0.954 ( $P<0.001$ for all comparisons), and sequence divergences ranged between 4 and $11 \%$. Given these findings and by the standards of genetic and ecological differentiation in other published species groups, $T$. tabaci must be considered a complex of cryptic (sub)species. Heredity (2004) 93, 364-370. doi:10.1038/sj.hdy.6800512

Published online 7 July 2004

Keywords: biotypes; cryptic species; host races; phylogeography; phytophagous insects; Thysanoptera

\section{Introduction}

Thrips comprise a single insect order with around 5000 described species. While most thrips species remain inconspicuous, some show all the features that predispose them to be major pest species, by causing direct feeding damage and by spreading viral diseases to food, fiber and ornamental crops. Most thrips are host-plant specific, but some economically important species are polyphagous. The diversity of ecosystems in which these latter species are encountered raises the question of genetic variation in the use of resources by different populations (reviewed by Futuyma and Peterson, 1985). From an evolutionary point of view, it is interesting to assess the genetic structure of thrips populations relative to their host plants. Moreover, pest management through the use of natural enemies or the application of specific pesticides would certainly benefit from the ability to discriminate between thrips genotypes on different hosts.

Thrips tabaci (Thysanoptera; Terebrantia) is a widespread pest that has attracted special interest as the first identified vector of tomato spotted wilt virus (TSWV), a tospovirus with a wide host range that is capable of causing serious epidemics and crop losses. Interestingly, the effectiveness of $T$. tabaci as a vector for TSWV and host-plant preference can vary dramatically among populations (eg Zawirska, 1976). Given this ecological

Correspondence: P Brunner, Agroscope FAW, Swiss Federal Research Station, PO Box 185, Wädenswil CH-8820, Switzerland.

E-mail: patrickcbrunner@netscape.net

Received 25 September 2003; accepted 24 April 2004; published online 7 July 2004 diversity, Zawirska (1976) suggested that T. tabaci consists of two biotypes. The 'tabaci type' is found on tobacco plants and is associated with the spread of TSWV. In contrast, populations of the 'communis type' infest a variety of host-plants (but not tobacco) and are not vectors for TSWV. These considerations, however, have received very little attention, and today $T$. tabaci is generally assumed to be a single, cosmopolitan and highly polyphagous species. Host-plant transfer experiments, and a survey of studies on the long-standing problem of vector status (Chatzivassiliou, 2002) support Zawirska, and reinforce the idea that $T$. tabaci is a heterogeneous taxon representing at least two differentiated biotypes or subspecies.

Resolution of the taxonomic status of $T$. tabaci populations has been hampered by a lack of population genetic studies. The development of molecular genetic techniques during the last two decades, particularly analysis of mitochondrial DNA (mtDNA), has substantially contributed to an understanding of natural genetic diversity and speciation issues (Moritz et al, 1987; Avise, 1994). These approaches are especially helpful in such puzzling groups as thrips, which show a mosaic of diverse ecological traits superimposed onto a conserved morphology. A growing appreciation of cryptic genetic differentiation in insects suggests three alternatives: $T$. tabaci might be (a) a single polyphagous species, (b) a complex of host races with partial genetic differentiation but ongoing gene flow, or (c) a complex of morphologically cryptic species no longer joined via gene flow.

This study investigates the evolutionary relationship of $T$. tabaci populations based on sequence variation of the mitochondrial cytochrome oxidase I (COI) gene. The 
goals are: (1) To examine (for the first time) the genetic population structure of $T$. tabaci over a broad range. Such comparisons are essential, otherwise previous and more restricted results cannot be placed within a more general framework and must therefore remain isolated and anecdotal. (2) To evaluate congruence of genetic findings with morphology (upon which current taxonomy is based), and ecological characteristics such as host-plant preference and vectoring efficiency in order to corroborate previous laboratory findings.

\section{Materials and methods}

\section{Data collection}

T. tabaci were collected on leek and tobacco plants from 22 sites in Switzerland, Greece and Bulgaria (Figure 1). Specimens were first identified morphologically, and only thrips unambiguously identified as $T$. tabaci were subjected to genetic analyses. In addition, morphological species status was confirmed by an external specialist (S Nakahara, USDA) for specimens assigned to the three major clades (T, L1 and L2) identified in Figures 2-4.

Total genomic DNA was extracted from single thrips using the slightly modified protocol by Kawasaki (1990), and a fragment of the mitochondrial COI gene was amplified via a standard PCR reaction using the primers C1-J-1751 and C1-N-2191 (Simon et al, 1994). DNA sequences were generated using the same two primers on an ABI 3100 automated sequencer (Applied Biosystems, Foster City, CA, USA). All DNA was sequenced in both directions and aligned with the multiple sequence editor CLUSTAL X (Thomson et al, 1997).

\section{Data analysis}

Genetic variability and pairwise (traditional) $F_{\text {ST }}$ statistics were calculated using ARLEQUIN version 2.0. (Schneider et al, 2000) to compare among group divergence with studies from other species.

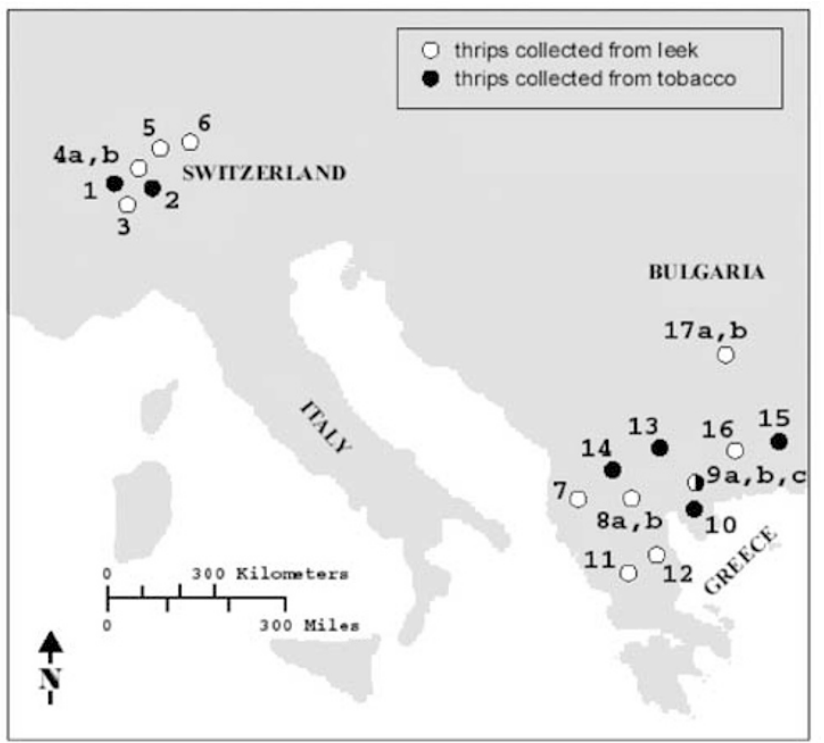

Figure 1 Map showing the sampling sites for T. tabaci: 1, Biel; 2, Murten; 3, Rütihof; 4, Wädenswil; 5, Zürich; 6, Trungen; 7, Florina; 8, Ionia; 9, Arethoussa; 10, Doumbia; 11, Karditsa; 12, Volos; 13, Gorgopi; 14, Pella; 15, Velkio; 16, Xanthi; 17, Bulgaria.

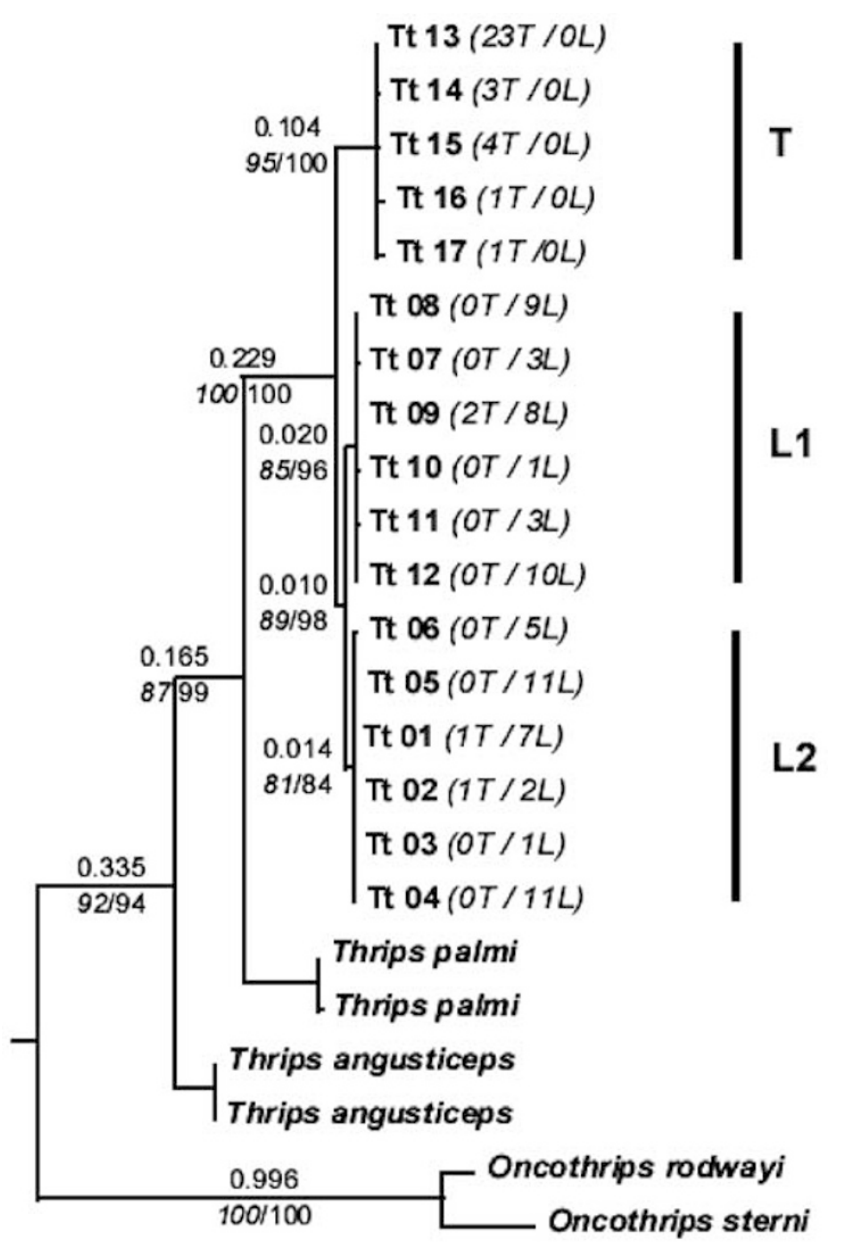

- 0.1 substitutions / site

Figure 2 Maximum-likelihood tree based on the TMV $+\mathrm{I}+\mathrm{G}$ evolutionary model. For clarity, branch length distances, and ML (italics) and MP bootstrap values are given only for major clades. Haplotype designation for T. tabaci (' $\mathrm{Tt}$ ') is as per Table 1, followed by the number of haplotypes detected on tobacco plants ' $\mathrm{T}$ ' or leek ' $\mathrm{L}$ ', respectively. The tree was rooted using the COI sequence of L. migratoria.

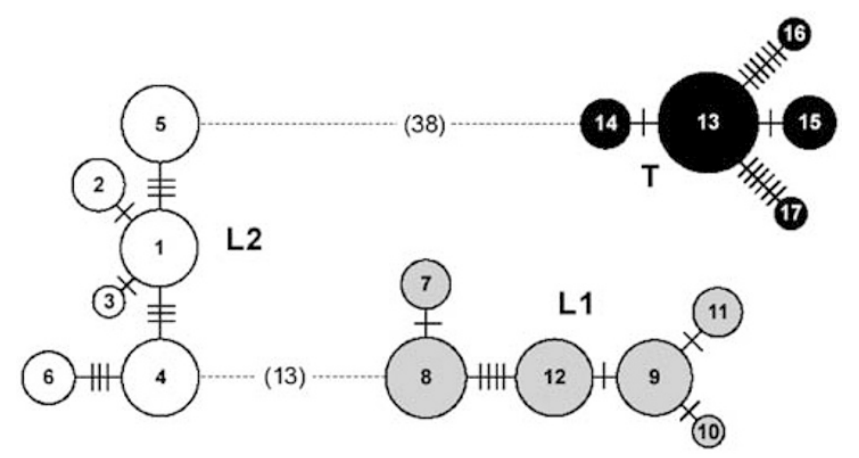

Figure 3 The estimated cladogram with 95\% plausible set of haplotype connections for T. tabaci. Numbers within circles are the haplotype designations as per Table 1 . Hash marks and numbers in parenthesis denote single-base substitutions and dashed lines indicate connections between divergent clades. Circle size is proportional to sample size and circle color indicates the three major clades also identified in Figures 2 and 4 (ie $\mathrm{T}=$ tobacco; L1, L2 = leek). 


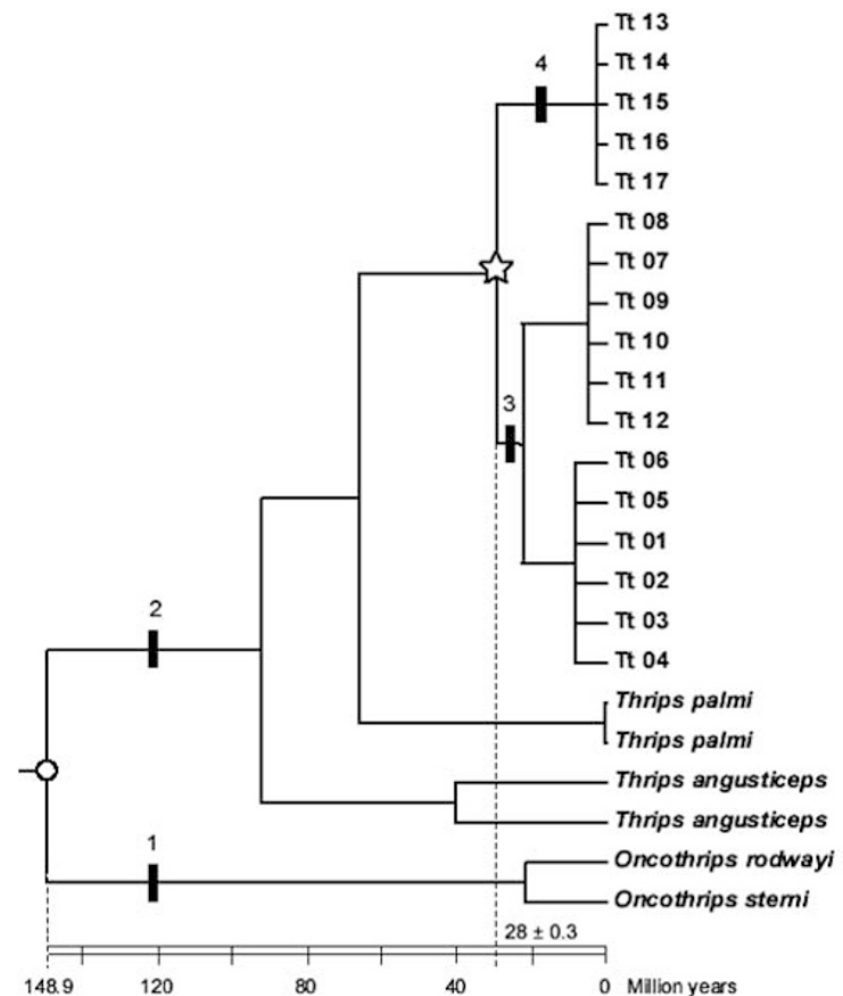

Figure 4 Ultrametric tree based on the NPRS. Black bars indicate major transitions during the evolution of the Thysanoptera: 1, suborder Tubulifera; 2 , suborder Terebrantia; 3, leek as the main host plant; 4 , tobacco as the main food plant. The scale bar below the tree shows the time scale resulting from a calibration of the molecular clock (fossil record of Thysanoptera) based on the circled node. The star indicates the node of the phylogenetic split of T. tabaci into tobacco- and leek-associated lineages.

We applied maximum-likelihood (ML) and maximum parsimony (MP) analyses using the computer program PAUP* 4.0b10 (Swofford, 2002) to assess phylogenetic relationships. MODELTEST 3.06 (Posada and Crandall, 1998) was used to determine the substitution model that best fits the data set for the ML analysis. The hierarchical likelihood-ratio test (LRT) implemented in MODELTEST selected the TVM $+\mathrm{I}+\mathrm{G}$ model (proportion of invariable sites $=0.39$; gamma distribution shape $=0.49$ ). The MP analysis using all equal weights was performed under the heuristic search option (50 replicate searches with random addition of taxa). A bootstrap analysis was performed to test for statistical significance of the trees generated with 500 pseudoreplicates and under the fast stepwise addition option for MP. Two specimens each from T. palmi and T. angusticeps served as intrageneric comparisons, and because they are considered closely related to $T$. tabaci (Brunner et al, 2002). GenBank sequences from Oncothrips sterni (AF386719) and $O$. rodwayi (AF386693) served as representatives for the Tubulifera, the other suborder of the Thysanoptera. Locusta migratoria (NC_001712) was used as an outgroup.

Gene genealogies within T. tabaci was also estimated using TCS (Clement et al, 2000), a program that implements the estimation of gene genealogies from DNA sequences as described by Templeton et al (1992). This cladogram estimation method - also known as statistical parsimony - displays the number of base-pair differences between haplotypes and provides the $95 \%$ parsimoniously plausible branch connection between haplotypes.

An LRT (Huelsenbeck and Crandall, 1997) was performed with and without a molecular clock enforced. To date, major cladogenetic events such as ultrametric trees were constructed using the nonparametric rate smoothing (NPRS) method (Sanderson, 1997) as implemented in TreeEdit 1.0 (Rambaut and Charleston, 2001). To estimate roughly divergence times between major clades, we applied a molecular clock using the estimated age of extant Thysanopterans (Mayhew, 2002). The age was estimated by first compiling the age of the oldest fossil definitely attributed to the Thysanoptera, from Ross and Jarzembowski (1993). Second, these taxon age estimates were modified by making a further logical step based on phylogenetic relationships. Sister taxa (ie the two suborders Tubulifera and Terebrantia) are, by definition, the same age. Therefore, if the estimated age of two sister taxa using oldest fossils differed, both were assigned the age of the oldest of the pair. This assumes that any inconsistency in the age of the earliest fossils arises from the incompleteness of the fossil record rather then through paraphyly. Finally, adding Oncothrips as a genetically distant comparison (eg Brunner et al, 2002) is conservative because it may underestimate the age of T. tabaci.

\section{Results}

\section{Phylogeny of T. tabaci}

COI sequencing yielded a $433 \mathrm{bp}$ long fragment for 107 individuals of T. tabaci. A total of $56 \mathrm{bp}$ (12.9\%) were polymorphic, and these defined 17 haplotypes (sequences deposited in GenBank with Accession nos. AY196831-AY196849). All variation was in the form of silent, single base-pair substitutions, except for position 46. Here, guanine $(G)$ is replaced by adenosine (A), resulting in an amino-acid replacement from glycine to serine (Table 1).

The ML tree revealed two major subdivisions that primarily arrayed $T$. tabaci haplotypes according to host plants: clade ' $\mathrm{T}$ ' was exclusively composed of haplotypes from $32 \mathrm{~T}$. tabaci individuals collected on tobacco from Greece, and the other clades L1 + L2 contained haplotypes from 75 individuals collected on leek with the exception of four individuals that were collected on tobacco (Figure 2). A second division was observed within the 'leek' clade: clade L1 was exclusively composed of individuals collected in Greece, while clade L2 was composed of all individuals collected in Switzerland (sites 1-6) plus individuals from locations 7 (Greece) and 17 (Bulgaria). The maximum parsimony tree (not shown) revealed, with stronger bootstrap values for the major clades, the same clustering as in the ML tree. The dominant feature of phylogenetic splits into three major clades (T, L1 and L2) is also reflected in the 95\% parsimoniously plausible haplotype network (Figure 3).

\section{Rates of evolution}

An LRT with $(-\ln L=1980.58)$ and without $(-\ln L=1997.81)$, the molecular clock enforced rejected overall constancy of the rates of evolution in the 
Table 1 Variable positions in the $433 \mathrm{bp}$ segment of the COI gene defining 17 different haplotypes and their frequency distribution across 22 sampling sites of Thrips tabaci

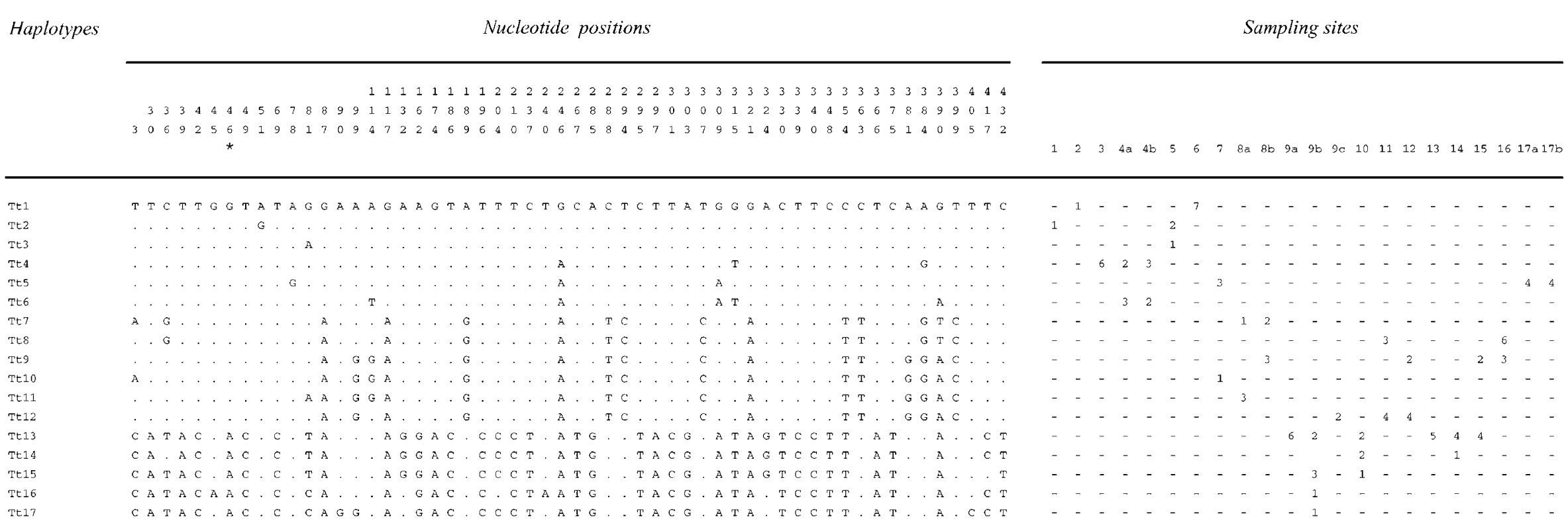

Amino-acid replacement substitutions are indicated by asterisks. 
Thysanoptera $(\delta=34.46, \mathrm{df}=21, P=<0.05)$. In the absence of rate constancy, we used the NPRS method to construct an ultrametric tree (Figure 4) based on the ML tree, which was used for further analyses. Calibration of a molecular clock with the estimated date of divergence of extant Thysanoptera (148.9 million years) allowed us to date roughly the main cladogenetic events that occurred in T. tabaci history. The initial divergence into the leek- and tobacco-associated clades (L1, L2 and T) occurred during the Oligocene-Miocene boundary (around 28 million years ago). Subsequent diversification of the leek-associated lineages into L1 and L2 occurred during the Miocene (around 21 million years ago; Figure 4).

\section{Discussion}

This study was designed primarily to test the hypothesis that $T$. tabaci represents a host-plant associated taxonomic complex. Our analyses clearly indicate that genetic differentiation is significant among (sympatric and allopatric) populations of $T$. tabaci collected from different host plants. Clustering analysis and haplotype networks strongly suggest three distinct, well-supported major lineages in T. tabaci. The many fixed nucleotide differences support both an ancient origin and a longterm isolation of these lineages (Table 1).

\section{Genetic findings and current taxonomy}

Traditionally, taxa are distinguished using morphological characters. However, not all species lend themselves to this approach, most often because of insufficient phenotypic variation. Thrips are notorious for eliciting taxonomic problems due to their minute size, a scarcity of solid morphological characters, the fact that most species are associated with more than one host, and the finding that different species often coexist on the same plant. Crespi et al (1998), for example, examined Australian gall-forming thrips species using sequences of the COI gene. They found that each species apparently represented a pair of sibling species, previously undistinguishable due to 'long-term morphological stasis'. Thus, the repeated appearance of stereotypic morphologies (and ecotypes) does not necessarily indicate sister relationships, perhaps as a consequence of the unique asymmetrical mouthparts that allow only for a piercingsucking type of feeding and, hence, strongly limit the possibilities of ecological and morphological diversification in this order (ie 'phylogenetic constraints' sensu; Douglas and Matthews, 1992; Douglas and Brunner, 2002). In fact, Zawirska (1976) noted that a character on the abdomen of the second larval stage of $T$. tabaci differentiates the two biotypes (ie 'tabaci' vs 'communis'), whereas adults are morphologically indistinguishable. Unfortunately, we were not capable of confirming (or refute) this observation before the submission of the manuscript.

Molecular evidence presented in this study strongly suggests T. tabaci forming three distinct, well-supported lineages consistent with a tobacco group and two leek groups. Given that tobacco and leek groups are genetically distinct, are they host races (ie lineages with partial reproductive isolation as a consequence of adaptation to different hosts)? Or are they instead cryptic species between which gene flow has ceased? Clearly, the answer to these questions depends on one's definition of species. However, the thrips populations studied here would qualify as (sub)species under the view of genetic distinctiveness in sympatry because the tobacco- and the two leek-associated lineages remain distinct both genetically and ecologically. Under the biological species concept, on the other hand, complete reproductive isolation is required before two groups can be accorded species status. Although estimates of $F_{\mathrm{ST}}$ alone cannot be used to conclude whether all gene flow between two groups has ceased (Whitlock and McCauley, 1999), it is useful to put them into the context of similar estimates for other groups. For example, estimates for host races of the gall-moth Gnorimoschema gallaesolidaginis (Nason et al, 2002) and the pea aphid Acyrthosiphon pisum (Via, 1999) are 0.16 and 0.21 , respectively. In contrast, estimates obtained for sympatric species of Gryllus (crickets) (Harrison, 1979) and (Echenopa) planthoppers (Guttman and Weigt, 1989) are 0.92 and 0.91, respectively. Differentiation between $T$. tabaci lineages clearly exceeds typical host-race estimates, and falls within the range for sympatric species; $F_{\mathrm{ST}}=0.824$ between clades L1 and L2, 0.946 between clades L1 and T and 0.954 between clades L2 and T. Finally, nucleotide sequence divergences between leek and tobacco haplotypes equate with hostspecific, morphologically indistinguishable sibling species of Australian gall-forming thrips (range 8-16\%; Crespi et al, 1998), but are substantially lower than those detected among morphologically distinguishable thrips species (range 16-27.5\%; Brunner et al, 2002).

Population structure and host-associated differentiation Whereas geographic isolation and genetic drift contribute to pronounced intraspecific phylogeographic structure, gene flow retards the genetic divergence of populations (Avise et al, 1987). The latter may be massive enough to reverse adaptive differentiation, unless the integrity of populations is maintained by reproductive isolation. In other known phytophagous host-race pairs (eg Eurosta, Abrahamson and Weis, 1997; Rhagoletis, Feder et al, 1998) common suites of factors such as strong host preference and/or mating on the host plant often contribute to the maintenance of host association and assortative mating. If host fidelity is perfect, then reproductive isolation is complete. If some migration between hosts occurs, then reproductive isolation can only evolve if there is premating selection against migrants or if there is some form of postmating selection against hybrid progeny (Liou and Price, 1994).

How reliable is host-plant choice in T. tabaci? Hostplant preference in T. tabaci seems to be so pronounced that the two differentiated lineages have been found in neighboring fields (sampling sites $9 \mathrm{~b}$ and $9 \mathrm{c}$ in Figure 1), but strictly on their respective host-plant type (Figure 2). This strongly suggests that there is an active choice for the right plant. However, genetic haplotype assignment revealed that host preference was not perfect as four adult females were collected on the 'wrong' host plant (Figure 2). One explanation is that these host-mismatched adult thrips accidentally migrated to the reciprocal host. Although thrips are weak flyers, their fringed wings enable them to remain easily airborne long enough to travel between neighboring fields, and to be blown by the wind over far greater distances as a 
component of 'air plankton' (Lewis, 1997). Few empirical data are available to determine the extent and cause of reproductive isolation between host-associated insect populations. One notable exception is the apple maggot fly, Rhagoletis pomonella. Here, host choice studies (apple and hawthorn, respectively) suggested that about $6 \%$ of adult $R$. pomonella on a given host may have migrated from the other host (Feder et al, 1994). Although this host mismatch is almost twice that found in leek vs tobacco populations of T. tabaci, selection on phenology mediated by different rates of fruit development and rot may be enough to eliminate any gene flow from this migration in R. pomonella (Feder et al, 1997). A second explanation is that barriers to gene flow other than host choice, such as selection against migrants or hybrid sterility, must also exist. Indeed, reciprocal laboratory host-plant experiments (Chatzivassiliou et al, 2002; Chatzivassiliou, 2002) suggests strong host-specific (physiological) adaptations. While $T$. tabaci populations from both host plants apparently thrived on leek, those originally collected from leek failed to survive on tobacco.

Implications for pest management strategy

Thrips continue to increase as major pests of agricultural and greenhouse crops. Although T. tabaci is damaging in its own right, its potential for harm is far greater because it can serve as a vector of TSWV. Virus diseases can be very difficult to control and usually hinge on the control of the vector. Control of thrips with insecticides, however, is difficult. Eggs within leaf tissue and pupae in the soil/leaf litter are protected from most sprays. Similarly, larvae and adults are difficult to contact with spray applications because they are highly agile and protected within developing buds and flowers. Despite these basic biological facts, many growers still utilize insecticides as their primary method of control. The situation has been exacerbated as the heavy pesticide usage has encouraged the rapid development of insecticide resistance in many species. As early as in the mid1950 s at least five insecticides tested were already becoming ineffective against T. tabaci, and new products rarely retain their efficacy for more than 4 years (Richardson and Wene, 1956). Furthermore, stringent quality requirements allow only minimum/zero pesticide residues and little or no damage on marketable produce. To combat these relatively recent problems, much effort has been devoted to developing integrated pest management (IPM) to control pests, including thrips. IPM is a complex approach to pest control with biological control techniques playing a crucial part. Hence, it is clear that an understanding of the biology, ecology, population structure, etc, of the pest - as herein presented for T. tabaci - is a fundamental first step to make sound decisions. For example, the identification of genetically and ecologically distinct $T$. tabaci lineages can substantially reduce the application of pesticides. While the occurrence of the vector lineage (ie clade ' $T$ '; the 'tabaci-type' sensu Zawirska) might still need the rapid application of specific pesticides to limit the spread of TSWV, this might be reduced in favor of biological control techniques such as predatory mites or bugs in case of the nonvector lineages 'L1' and 'L2' (the 'communis type'). Additional experiments with different plants remain to be conducted, but eventually our knowledge of host-plant preference/avoidance will allow growers to slow the development of $T$. tabaci (and other thrips) populations and even break the life cycle by careful selection of plants and cultivars.

To conclude, genetic studies of apparently generalist phytophagous insects often reveal complexes of genetically differentiated host races or cryptic species. The molecular results presented in this study, and previous observations and experiments on the ability to transmit TSWV and host preference, provide independent and very strong evidence that $T$. tabaci represents a complex of at least three taxa. We were able to demonstrate unexpectedly strong genetic differentiation among T. tabaci populations that suggests an ancient origin for the three major phylogenetic lineages. Thus, our findings clearly refute the general belief that $T$. tabaci is a single cosmopolitan and polyphagous species. On the contrary, by the standards of genetic and ecological differentiation in other species groups, the recognition of hostassociated and distinct $T$. tabaci (sub)species must be considered.

\section{Acknowledgements}

We thank B Frey and F Schwaller for providing invaluable help in the lab, and constructive comments from JFW Brookfield, ME Douglas and an anonymous referee improved earlier versions of this manuscript. We are also grateful to $S$ Nakahara (Systematic Entomology Lab, USDA/ARS, USA) for thrips identification, and D Peters (Department of Virology, Agricultural University of Wageningen, The Netherlands) for providing T. tabaci samples. This study was supported by Swiss National Science Foundation Grant 3100-064045.00 to PCB and JEF and a Greek Scholarship Foundation Grant to EKC.

\section{References}

Abrahamson WG, Weis AE (1997). Evolutionary Ecology Across Three Trophic Levels: Goldenrods, Gallmakers, and Natural Enemies. Princeton University Press: Princeton, NJ.

Avise JC (1994). Molecular Markers, Natural History and Evolution. Chapman \& Hall: New York.

Avise JC, Arnold J, Ball MR, Bermingham E, Lamb T, Neigel JE et al (1987). Intraspecific phylogeography: the mitochondrial bridge between population genetics and systematics. Ann Rev Ecol Systemat 18: 489-522.

Brunner PC, Fleming C, Frey JE (2002). A molecular identification key for economically important thrips species (Thysanoptera: Thripidae) using direct sequencing and a PCRRFLP-based approach. Agric Forest Entomol 4: 127-136.

Chatzivassiliou EK (2002). Thrips tabaci: an ambiguous vector of TSWV in perspective. In: Marullo R, Mound L (eds) Thrips and Tospoviruses: 7th International Symposium on Thysanoptera. Australian National Insect Collection: Canberra, Australia, pp 69-75.

Chatzivassiliou EK, Peters D, Katis NI (2002). The efficiency by which Thrips tabaci populations transmit tomato spotted wilt virus depends on their host preference and reproductive strategy. Phytopathology 92: 603-609.

Clement M, Posada D, Crandall KA (2000). TCS: a computer program to estimate gene genealogies. Mol Ecol 9: 1657-1659.

Crespi BJ, Carmean DA, Mound LA, Worobey M, Morris D (1998). Phylogenetics of social behavior in Australian gall-forming thrips: evidence from mitochondrial DNA sequences, adult morphology and behavior, and gall morphology. Mol Phylogenet Evol 9: 163-180. 
Douglas ME, Matthews WJ (1992). Does morphology predict ecology? Hypothesis testing within a freshwater stream fish assemblage. Oikos 65: 213-224.

Douglas MR, Brunner PC (2002). Biodiversity of central alpine Coregonus (Salmoniformes): impact of one-hundred years of management. Ecol Appl 12: 154-172.

Feder JL, Berlocher SH, Opp SB (1998). Sympatric host-race formation and speciation in Rhagoletis (Diptera: Tephritidae): a tale of two species for Charles D. In: Mopper S, Strauss SY (eds) Genetic Structure and Local Adaptation in Natural Insect Populations. Chapman \& Hall: New York, pp 408-441.

Feder JL, Opp S, Wlazlo B, Reynold K, Go W, Spizak S (1994). Host fidelity as an effective premating barrier between sympatric races of the apple maggot fly. Proc Natl Acad Sci USA 94: 11417-11421.

Feder JL, Stolz U, Lewis K (1997). The effects of winter length on the genetics of apple and hawthorn races of Rhagoletis pomonella (Diptera: Tephritidae). Evolution 51: 1862-1876.

Futuyma DJ, Peterson SC (1985). Genetic variation in the use of resources by insects. Annu Rev Entomol 30: 217-238.

Guttman SI, Weigt LA (1989). Macrogeographic genetic variation in the Echenopa binotata complex (Homoptera: Membracidae). Ann Entomol Soc Am 82: 1156-1165.

Harrison RG (1979). Speciation in North American field crickets: evidence from electrophoretic comparisons. Evolution 33: 1009-1023.

Huelsenbeck JP, Crandall KA (1997). Phylogeny estimation and hypothesis testing using maximum likelihood. Annu Rev Ecol Syst 28: 437-466.

Kawasaki ES (1990). Sample preparation from blood, cells, and other fluids. In: Innis MA, Gelfand DH, Sninsky JJ, White TL (eds) PCR Protocols. A Guide to Methods and Applications. Academic Press: San Diego, pp 146-152.

Lewis T (1997). Flight and dispersal. In: Lewis T (ed) Thrips as Crop Pests. University Press: Cambridge, MA, pp 175-196.

Liou LW, Price TD (1994). Speciation by reinforcement of premating isolation. Evolution 48: 1451-1459.

Mayhew PJ (2002). Shifts in hexapod diversification and what Haldane could have said. Proc R Soc Lond Ser B 269: 969-974.

Moritz C, Dowling TE, Brown WM (1987). Evolution of animal mitochondrial DNA: relevance for population biology and systematics. Annu Rev Ecol Syst 18: 269-292.

Nason JD, Heard SB, Williams FR (2002). Host-associated genetic differentiated in the goldenrod elliptical-gall moth,
Gnorimoschema gallaesolidaginis (Lepidoptera: Gelechiidae). Evolution 56: 1475-1488.

Posada D, Crandall KA (1998). Modeltest: testing the model of DNA substitution. Bioinformatics 14: 817-818.

Rambaut A, Charleston M (2001). TreeEdit. Ver 2.0. Department of Zoology, University of Oxford: UK.

Richardson BH, Wene GP (1956). Control of onion thripsand its tolerance to certain chlorinated hydrocarbons. J Econ Entomol 49: 333-335.

Ross AJ, Jarzembowski EA (1993). Arthropoda (Hexapoda; Insecta). In: Benton MJ (ed) The Fossil Record 2. Chapman \& Hall: London, pp 363-426.

Sanderson M (1997). A nonparametric approach to estimate divergence times in the absence of rate constancy. Mol Biol Evol 14: 1218-1231.

Schneider S, Roessli D, Excoffier L (2000). Arlequin: A Software for Population Genetics Data Analysis. Ver 2.000. Genetics and Biometry Lab, Department of Anthropology, University of Geneva: Geneva.

Simon C, Frati F, Beckenbach A, Crespi B, Liu H, Flook P (1994). Evolution, weighing, and phylogenetic utility of mitochondrial gene sequences and a compilation of conserved polymerase chain reaction primers. Ann Entomol Soc Am 87: 651-701.

Swofford DL (2002). PAUP*. Phylogenetic Analysis Using Parsimony (* and other methods). Version $4.0 \mathrm{~b} 10$ Sinauer: Sunderland, MA.

Templeton AR, Crandall KA, Sing CF (1992). A cladistic analysis of phenotypic associations with haplotypes inferred from restriction endonuclease mapping and DNA sequence data. III. Cladogram estimation. Genetics 132: 619-633.

Thomson JD, Gibons TJ, Plewniak F, Jeanmougin F, Higgins DG (1997). The ClustalX windows interface: flexible strategies for multiple sequence alignment aided by quality analysis tools. Nucleic Acids Res 24: 4876-4882.

Via S (1999). Reproductive isolation between sympatric races of pea aphids. I. Gene flow restriction and habitat choice. Evolution 53: 1446-1457.

Whitlock MC, McCauley DE (1999). Indirect measures of gene flow and migration $F_{\mathrm{ST}} 1 /(4 \mathrm{Nm}+1)$. Heredity 82 : 117-125.

Zawirska I (1976). Untersuchungen über zwei biologische Typen von Thrips tabaci Lind. Thysanoptera, Thripidae) in der VR Polen. Arch Phytopathol Pflanzenschutz 12: 411-422. 